\title{
Leukotrienes and sex: strange bedfellows?
}

\author{
Lewis J. Smith
}

Department of Medicine, Northwestern University, Chicago, Illinois, USA

\begin{abstract}
Leukotrienes are proinflammatory lipid mediators that have been shown to be upregulated in several diseases, including asthma, aspirinexacerbated respiratory disease (AERD), inflammatory bowel disease, and acute respiratory distress syndrome. Leukotrienes have been explored as therapeutic targets for these diseases and others; however, leukotriene inhibitors have had limited success in the clinic. There are noted differences in the incidence of leukotriene-mediated diseases in males and females, but sex as a factor in the response to leukotriene inhibitors has not been fully explored. In this issue of the JCI, Pace and colleagues present evidence that there are sex-specific differences in the effectiveness of certain leukotriene inhibitors and link the differences in response to the presence of androgens. The results of this study indicate that sex needs to be taken into consideration in the future evaluation of leukotriene inhibitors to treat disease.
\end{abstract}

\section{Leukotrienes and disease}

Leukotrienes are bioactive mediators that were initially identified in the 1970s, and the biosynthetic pathways involved in leukotriene formation were elucidated around the same time (1). The major leukotrienes were designated as leukotriene $\mathrm{B}_{4}\left(\mathrm{LTB}_{4}\right)$ and as the cysteinyl leukotrienes $\left(\mathrm{LTC}_{4}, \mathrm{LTD}_{4}\right.$, and $\left.\mathrm{LTE}_{4}\right)$. Subsequently, several leukotriene receptors (BLT $_{1}$, $\mathrm{BLT}_{2}, \mathrm{CysLT}_{1}, \mathrm{CysLT}_{2}$ ) were discovered, and small molecules were developed that inhibited leukotriene synthesis either by targeting 5-lipoxygenase (5-LO), 5-LOactivating protein (FLAP), cytosolic phospholipase $\mathrm{A}_{2}\left(\mathrm{cPLA}_{2}\right)$, or molecules, such as leukotriene receptor antagonists, that interfere with the ability of leukotrienes to mediate their effects. Increased leukotriene production has been linked to several inflammatory diseases through a series of animal, in vitro, and human studies in which leukotriene levels were measured under basal and stimulated conditions and in which the newly developed leukotriene modifiers were administered to various animal species, healthy individuals, and patients with disease. Specifically, leukotriene expression has been associated with asthma $(2,3)$, aspirin-exacerbated respiratory disease (AERD) (4), allergic rhinitis (5), inflammatory bowel disease (6), and acute respiratory distress syndrome (7). However, with rare exceptions, such as AERD (8), leukotriene modifiers have shown only a modest beneficial effect on diseases in which leukotrienes would be expected to play an important role. Several explanations, including differences in leukotriene levels in individual patients, the heterogeneity of disease phenotypes, and differences in drug pharmacokinetics, pharmacodynamics, and pharmacogenomics, have been proposed for why targeting leukotrienes does not benefit leukotriene-related diseases (9); however, there has not been a clear explanation for why antileukotriene drugs have not worked as expected.

\section{Sex and the response to leukotriene inhibition}

One possible explanation comes from the study of diseases associated with elevat-

Related Article: p. 3167

Conflict of interest: The author has declared that no conflict of interest exists.

Reference information: J Clin Invest. 2017;127(8):2895-2896. https://doi.org/10.1172/JCI95717. 


\section{Conclusions and future directions}

This report by Pace and colleagues provides new insights into the influence of sex (and sex hormones) on leukotriene synthesis and its inhibition. While the potential of these results to be of clinical relevance is exciting, additional preclinical and clinical studies are needed to define the true clinical impact, which is likely to be complex. For example, Pace et al. focus primarily on inflammatory processes and leukocytes that predominantly generate $\mathrm{LTB}_{4}$. Although a number of inflammatory diseases, including scleroderma lung disease (13), inflammatory bowel disease (6), sickle cell disease (14), and cardiovascular disease (15), are reported to be associated with increased $\mathrm{LTB}_{4}$ levels, there are few, if any, examples in which treatment of these diseases with leukotriene synthesis inhibitors has shown benefit. The diseases in which there is the most information relevant to leukotriene biology are asthma, AERD, and allergic rhinitis, to which cysteinyl leukotrienes are known to contribute. For example, well-characterized mouse models of asthma have been used to study the role of leukotrienes and leukotriene modifiers, including cysteinyl leukotriene receptor blockers, which have become widely used in clinical practice $(16,17)$. Experiments with disease models that have a demonstrated response to leukotriene modification could be used to further explore sex-dependent differences in cysteinyl leukotriene synthesis and the response to leukotriene synthesis inhibitors and receptor blockers. Additionally, sufficient numbers of human peripheral blood eosinophils could be obtained from individuals with elevated eosinophils counts and used to determine whether or not there are sex-specific differences in stimulus-generated cysteinyl leukotriene levels in vitro and/or in the ability of leukotriene modifiers to block these effects.

Retrospective analyses of databases from studies performed in the 1990s, which tested leukotriene synthesis inhibitors and receptor antagonists in patients with asth- ma, may also provide further information on the role of sex and the response to leukotriene modification. These studies, which enrolled males and females, were of sufficient size to identify sex-specific differences. It is a bit surprising that a sex-dependent effect on response was not noted, considering the extensive post hoc analyses that are typically performed on such clinical trials. It is possible that earlier studies of leukotriene modifiers used doses that did not fully inhibit leukotriene synthesis. There is a recent interest in using higher doses of leukotriene modifiers and in delivering these inhibitors directly to the organ of interest so that high local concentrations are achieved. These approaches have the potential to be taken advantage of for possible identification of sex-dependent effects that have not previously been seen. For any new study, the influence of sex should be a predefined variable and the statistical analysis powered to identify such an effect. If the clinical studies identify a sex-specific effect on leukotriene synthesis inhibitors, it would validate the results of Pace and colleagues, expand the number of drugs whose effects are influenced by sex (18), and possibly lead to sex-specific therapy of a disease that affects large numbers of men and women.

\section{Acknowledgments}

I thank Peter Sporn for his thoughtful review and comments.

Address correspondence to: Lewis J. Smith, Northwestern University Feinberg School of Medicine, 750 N. Lake Shore Drive, Suite 707, Chicago, Illinois 60611, USA. Phone: 312.503.0501; Email: ljsmith@ northwestern.edu.

1. Samuelsson B. The discovery of the leukotrienes. Am J Respir Crit Care Med. 2000;161(2 Pt 2):S2-S6.

2. Smith LJ, Patterson R, Kern R, Krell R, Bernstein P. Effect of inhaled leukotriene D4 on human airways. Ann N Y Acad Sci.1988;524:298-306.

3. Spector SL, Smith LJ, Glass M. Effects of 6 weeks of therapy with oral doses of ICI 204,219, a leukotriene D4 receptor antagonist, in subjects with bronchial asthma. ACCOLATE Asthma Trialists Group. Am J Respir Crit Care Med.
1994;150(3):618-623.

4. Szczeklik A, et al. Bronchial aspirin challenge causes specific eicosanoid response in aspirinsensitive asthmatics. Am J Respir Crit Care Med. 1996;154(6 Pt 1):1608-1614.

5. Peters-Golden M, Gleason MM, Togias A. Cysteinyl leukotrienes: multi-functional mediators in allergic rhinitis. Clin Exp Allergy. 2006;36(6):689-703.

6. Rask-Madsen J, Bukhave K, Laursen LS, Lauritsen K. 5-Lipoxygenase inhibitors for the treatment of inflammatory bowel disease. Agents Actions. 1992;Spec No:C37-C46.

7. Masclans JR, et al. Possible prognostic value of leukotriene $\mathrm{B}(4)$ in acute respiratory distress syndrome. Respir Care. 2007;52(12):1695-1700.

8. Dahlén SE, et al. Improvement of aspirinintolerant asthma by montelukast, a leukotriene antagonist: a randomized, double-blind, placebocontrolled trial. Am J Respir Crit Care Med. 2002;165(1):9-14.

9. Drazen JM, et al. Pharmacogenetic association between ALOX5 promoter genotype and the response to anti-asthma treatment. Nat Genet. 1999;22(2):168-170

10. Postma DS. Gender differences in asthma development and progression. Gend Med. 2007;4(Suppl B):S133-S146.

11. Pace $S$, et al. Androgen-mediated sex bias impairs efficiency of leukotriene biosynthesis inhibitors in males. JClin Invest. 2017;127(8):3167-3176.

12. Pergola C, et al. ERK-mediated regulation of leukotriene biosynthesis by androgens: a molecular basis for gender differences in inflammation and asthma. Proc Natl Acad Sci U S A. 2008;105(50):19881-19886

13. Kowal-Bielecka O, et al. Elevated levels of leukotriene B4 and leukotriene E4 in bronchoalveolar lavage fluid from patients with scleroderma lung disease. Arthritis Rheum. 2003;48(6):1639-1646.

14. Jennings JE, et al. Elevated urinary leukotriene E4 levels are associated with hospitalization for pain in children with sickle cell disease. Am J Hematol. 2008;83(8):640-643.

15. Hoxha M, Rovati GE, Cavanillas AB. The leukotriene receptor antagonist montelukast its possible role in the cardiovascular field. Eur J Clin Pharmacol. 2017;73(7):799.

16. Miyahara N, et al. Leukotriene B4 release from mast cells in IgE-mediated airway hyperresponsiveness and inflammation. Am J Respir Cell Mol Biol. 2009;40(6):672-682.

17. Eiymo Mwa Mpollo MS, et al. Placenta growth factor augments airway hyperresponsiveness via leukotrienes and IL-13. JClin Invest. 2016;126(2):571-584

18. Legato MJ, Johnson PA, Manson JE. Consideration of sex differences in medicine to improve health care and patient outcomes. JAMA. 2016;316(18):1865-1866. 